\title{
Geo-referenced Open Data and Augmented Interactive Geo-visualizations as Catalysts for Citizen Engagement
}

\author{
Thore Fechner, Christian Kray \\ Institute for Geoinformatics, Westfälische Wilhelms-Universität Münster, Heisenbergstraße 2, 48149 Münster, Germany; \\ t.fechner@uni-muenster.de; c.kray@uni-muenster.de
}

\begin{abstract}
Citizen engagement figures prominently on political agendas around the world. In this context, high hopes are pinned to Open Government, open data and ICT tools. At the same time, there are fears of a widening digital divide, where large groups of society are in danger of being excluded from societal processes, for example due to having difficulties in using the online tools provided. In this paper, we propose an approach that has the potential to address many key issues in this context (e.g. accessibility, complexity, engagement). It relies on space and time as common integrators, and uses augmented interactive geo-visualizations to facilitate citizen engagement. We report on key challenges that need to be overcome to realize this approach and on initial progress towards this goal. We describe a set of prototypical tools aimed at supporting citizen engagement in the envisioned way, and discuss the approach as well as its potentials, issues and challenges in detail. Initial experiences and results indicate that our approach is not only technically feasible but it can also empower citizens to more effectively engage with societal and governmental processes.
\end{abstract}

Keywords: Open Government Data, Geo-visualization, Cartography, Maps, Collaboration, Space and Time, Engagement

Acknowledgement: We would like to acknowledge the work of Dennis Wilhelm, Gerald Pape and René Unrau, who were instrumental in realizing the tools reported on in this paper. Furthermore, we would like to extend our thanks to con terra and Esri Germany for their financial and other support.

$\mathrm{T}$

he digital era brings about new challenges and opportunities for public administrations worldwide. Accelerating globalization, changing communication habits and on-demand access to the Internet reshape society in many ways. In times of increasing mistrust against political elites, disenchantment with politics and a globalization of political processes, there is a general demand to put the relationship between government and citizens on a new footing. Consequently, calls for more transparency of government processes and decisions as well as for more civic participation coincide with demands for the reformation of state administrations that are perceived as old-fashioned and outdated. The concept of Open Government - the modernization of administrations in order to make them more transparent, accountable and to foster civic participation - plays a key role in this context. It differs from eGovernment in the sense that it is not just about the usage of Information and Communication Technologies (ICTs) for better public service delivery but instead focuses on transparent and open processes while engaging the public in government affairs (UN DESA and DPADM 2013, p. 45). Public engagement can take various forms such as public communication, public consultation or public participation (Rowe \& Frewer, 2005) that each require different kinds of information flows, stages and presentations. A key challenge is thus to provide and find the right formats and media for the different public engagement forms that actually attract citizens.

Open Government is seen as an "opportunity for government to redefine its role in society and help launch a new era of participatory government" (Tapscott, 2010, p. XVI). The goal is to allow citizens, non-profit organizations and the private sector to actively take part in the governing process (Tapscott, 2010, p. XVI). From a democratic theory point of view the concept of civil society is deeply associated with civic engagement and participation (Cohen \& Arato, 1994; De 
Tocqueville, 1985). "Civil society" can be defined in different ways, e.g. depending on various factors such as legal and organizational form, target group or voluntary engagement. We use it here as an umbrella term subsuming more structured Civil Society Organizations (CSOs) like NonGovernmental Organizations (NGOs) as well as loosely coupled interest groups or individuals.

In this context, Open Government Data (OGD) - the provision of government data to third parties for further use (Barnickel \& Klessmann, 2012, p. 129) - is often seen as a key enabler for the concept of Open Government. A key advantage of modern technology compared to analogue forms is that it greatly simplifies data provision, i.e via the cost-efficient release of exhaustive digital data related to government actions and decisions. OGD can also help to combat the widespread mistrust in governments and institutions - providing an opportunity to answer the presistent demand for transparency and information of civil society. At the same time OGD opens up new ways to satisfy increasing calls for extended citizen participation (Maier-Rabler \& Huber, 2011).

Even though citizen engagement varies according to the context, program or policy (Sheedy, MacKinnon, Pitre, \& Watling, 2008, p. 1), it always requires a certain form of communication. As OGD is meant to be primary data (i.e. not aggregated and processed) in order to maximize its usefulness and minimize bias, it needs to be contextualized and presented in an engaging and understandable way. Without proper presentations of OGD, Open Government Initiatives (OGIs) are likely to fail in their goal to engage citizens in government processes as the general public is unlikely to interpret and analyze raw data. Most OGls and advocates seem to be aware of this situation: frequently such initiatives include third parties from the public or private sector that act as intermediaries (Robinson, Yu, Zeller, \& Felten, 2009; UN DESA and DPADM, 2013).

One important and ubiquitous way of contextualizing data are maps. They are omnipresent on smartphones, accessible from the web and on public displays, and they are used e.g. to provide general information about the immediate surroundings, for navigation or to support specific tasks such as finding a nearby restaurant. New interaction possibilities have also emerged: once purely static maps have evolved into interactive, explorable and customizable "windows" to the world. Since space and place are universal concepts underpinning societies (from local and communal levels like neighborhoods, districts and cities to global levels like states and countries), maps are a natural fit for communicating and providing context to OGD. People are inherently familiar with space and time. Accessible geo-visualizations that are interactive and integrate open (government) data could thus empower citizens to voice their wishes, comments and critiques more effectivley while transforming the engagement process.

In this article, we therefore explore the potential, challenges and benefits of using space and time as integrators of open data and as facilitators in citizen engagement. Based on this approach, we propose to use augmented interactive geo-visualizations as an interface for citizen engagement. The remainder of this article is structured as follows: Section 1 discusses related work in the field of OGD, civil society and citizen participation. In section 2, we introduce our approach and the nessary steps to use geo-visualizations as facilitor in the OGD. Then, we report on first hand experiences with geospatial OGD in Germany (section 3), and we present first results on how to bridge the gap between the provision and use of geospatial OGD. In section 4, we outline three ways of how space, time and geo-visualizations can support citizen engagement. A discussion of the associated challenges, opportunities and limitations is conducted in the following section. Section 6 summarizes the main contributions of this article.

\footnotetext{
${ }^{1}$ see http://opengovdata.org/; the other principles are: complete, timely, machine processable, non-discriminatory, nonproprietary, accessible \& license-free.
} 


\section{Open Government (Data) and Citizen Participation}

OGD is an emerging field, and this section provides a short introduction to Open Government and then proceeds to reviewing the concept of civil society and its role in this context. We conclude by a concise look at research on the concept of participation, engagement and collaboration.

\section{Open Government and Public Administrations' Need for Innovation}

Public administration - here referred to as a set of institutions, persons and processes carrying out public service - has always played a crucial role in the (open) government process as it is said to "deliver democracy" (Hamilton, 2007, p. 14) and maintain citizen support of democracy (Ariely, 2013). According to Hamilton (2007, p. 18) our present democratic regimes need to have strong, technically competent, effective and efficient administrations in order to survive.

Paradoxically, many people feel that the existence of public service in a democracy contradicts the notion of government by the people (Ariely, 2013, p. 747). This paradox of public administration being both necessary and contentious in democratic regimes (Hamilton, 2007, p. $7 \mathrm{ff}$ ) has been a steady source of criticism and spurred the debate about the use and power of state administrations in democratic regimes. At the same time citizens' expectations are increasing with respect to transparency, participation and information. They increasingly wish to further participate, control, and understand decision-making processes and policies, and perceive government as a platform instead of a strict hierarchy with centralized decisions and structures (Eaves, 2010, p. 141). These circumstances lead to a strong pressure for governments to innovate and seek renewal.

In 2009, after his social media based election campaign, US President Barack Obama expressed his intention to increase transparency, participation and collaboration (Sifry, 2010, p. 117) leading to the Open Government Directive (Orszag, 2009).

The idea of Open Government itself is not new. One of the earliest written occurrences dates back to an article by Parks (1957), which sparked a long campaign that led to the final passage of the Freedom of Information Act in the US in 1966 (Yu \& Robinson, 2012, p. 185f). Later the term was used more as a synonym for government accountability, transparency and enhanced citizen engagement and participation ${ }^{2}$. A central component of Open Government is OGD. It refers to the free and exhaustive provision of government data to third parties for further use, which is facilitated by technological advances (Barnickel \& Klessmann, 2012, p. 129). Advocates claim that "government information is a form of infrastructure, no less important to our modern life than our roads, electrical grid, or water systems" (Malamud, 2010, p. 47).

Practically, the notion of OGD ties in with debates and developments emerging in the context of information technology and computer sciences in general. The Open Access movement for scientific literature or copy left license types such as Creative Commons or the Open Database License (ODbL) aim for easier reuse, increased collaboration and better innovation. Open technology standards have been and are being created by organizations such as the World Wide Web Consortium (W3C) or the Open Geospatial Consortium (OGC), which specify most of the World Wide Web's interfaces and standards. Advocates and practitioners consider OGD as essential for the realization of Open Government as the production and provision of transparent and re-useable data has the potential to institutionalize openness and to create commitment (Weinstein \& Goldstein, 2012).

This idea has gained traction in recent times: In 2011, the US and eight other states jointly launched the Open Government Partnership (OGP). Countries signing the declaration commit to support civic participation, to fight corruption and to pro-actively provide high value information including raw data that is easy to locate, understand, and use. Today, the number of undersigning

\footnotetext{
${ }^{2}$ The term even reached mass media in 1980. The first episode of "Yes, Minister" - a satirical British television show produced by the BBC - is entitled "Open Government".
} 
governments has risen to over $65^{3}$, and this number is continuously growing as public open data policies advance and are implemented, e.g. by the European Union member states (see the report of the European Parliament, 2011).

At the same time the debate about OGD is not restricted to practitioners - researchers are starting to enrich the debate from an interdisciplinary perspective (see Lathrop \& Ruma, 2010; Maier-Rabler \& Huber, 2011; Meijer, Curtin, \& Hillebrandt, 2012). In the controversial discussion about best practices and unexploited potentials policymakers and practitioners have started to recognize the importance of third parties for the success of OGD: NGOs, individual open data activists as well as private enterprises are intermediary actors promoting, monitoring and communicating the idea of OGD to the broader population (Yu \& Robinson, 2012).

On the whole, there are thus high expectations of Open Government (Nam, 2012, p. 349) but several issues such as privacy (Jonas \& Harper, 2010) are just emerging and will require further investigation.

\section{Civil Society}

Civil society can be defined in different ways, e.g. based on its organizational or legal form, clientele and target group, degree of philanthropy or voluntary engagement. For this article a broad definition and focus on all kinds of civil society actors is adopted - ranging from more organized CSOs such as NGOs to courageous individuals and internet activists that address specific goals.

A pragmatic definition of civil society is that of a sphere between the market, the state, and the family (Kocka, 2004). However, from a democratic theory point of view the term is also deeply associated with the values of democracy, participation, and civic engagement (see Almond \& Verba, 1963; Cohen \& Arato, 1994; De Tocqueville, 1985). In democratic regimes civil society plays a crucial part in citizen information and education. It acts as a "watchdog" over state actions, raises issues relevant to society, or protects minorities' rights and representation. Besides, it fulfills an intermediate function by linking and mediating between the state apparatus and the general population. It can also serve as a service provider in welfare states (Eikenberry, 2007; Pollack, 2003). In addition, civil society traditionally has a great share in providing and fostering innovations and emerging topics. A prominent example for such an influential actor of civil society in the field of OGD is the Open Knowledge Foundation (OKFN). Founded in Cambridge in 2004, it is specifically dedicated to promoting Open Data and Open Content in all their forms.

Klessmann et al. (2012) identify the following challenges civil society actors might face in the context of OGD. Firstly, the provision of OGD to the general public may challenge some organizations' supremacy as exclusive information sources and gatekeeper. Secondly, insufficient expertise of different civil society actors to handle data may lead to differences between organizations, as it favors only those technically capable and trained for working with OGD. Thirdly, civil society has to be careful in the adaptation of state tasks in its role as an intermediary. After all, the promotion and implementation of OGD is a joint task (Klessmann et al., 2012, p. 59f).

In addition to these challenges there are also questions related to the multiple roles civil society can take in the field of OGD. In particular, these concern its share in the implementation and use of OGD and are linked to general doubts about the trustworthiness and benevolence of CSOs and their influence on government decisions (Goodsell, 2006, p. 626). An often cited argument is that civil society is not always benevolent, philanthropic or pro-democratic (see Berman, 1997; Bob, 2012; Chambers \& Kopstein, 2001; Roth, 2003). Consequently, civil society should not carelessly be assigned state duties and responsibilities. Nevertheless, it has been recognized that a high degree of CSO involvement has often led to stronger processes and outcomes (Krafchik, 2013).

\footnotetext{
${ }^{3}$ http://www.opengovpartnership.org/
} 


\section{Participation, Collaboration \& Engagement}

Participation research has been approached from several directions: Social and political participation focuses on strengthening or enhancing participation, while the concept of citizenship looks more on rights and responsibilities (Cornwall \& Gaventa, 2000; Gaventa, 2002). Reflections on deliberative democratic theory examine the benefits of public disclosure or citizen engagement (Carpini, Cook, \& Jacobs, 2004).

Participation, collaboration or engagement are often mentioned together and used somewhat imprecisely and interchangeably throughout the literature. One of the earliest and well-known typologies for different levels of participation was provided by Arnstein (1969). He created the citizen participation ladder - describing eight different steps ranging from nonparticipation to different degrees of tokenism to citizen power. The concept of a ladder - describing increasing responsibility and distinctions in the information flow - is used to this day, for example to assess eParticipation systems (Steinmann, Krek, \& Blasche, 2004).

In an effort to overcome the imprecise usage of participation and engagement Rowe \& Frewer (2005) created a "working model" typology. It defines public engagement as consisting of public communication, public consultation and public participation. The three different types are distinguished based on the flow of information between participants and sponsors. Rowe \& Frewer (2005, p. 261) differentiate between participation and nonparticipation (public communication \& public consultation). They reduce the available classes in order to identify key mechanisms within each type, as they recognize that there are a many different forms within each category. Rowe \& Frewer (2005) are not looking at forms of collaboration, a vital aspect in Open Government.

We concur with Maier-Rabler \& Huber (2011) and Geiger \& Von Lucke (2012) that within OGls public administrations and citizens / civil society have to share responsibilities and to collaborate. This means, for example, that citizens should be consulted but more importantly that they should be allowed to have an actual effect on the result via shared editing and decision-making processes in (a)synchronous work models. As such and in summary the term citizen engagement is understood here as the idea to include civil society in the process of governance by engaging with it on different levels to enable it to have an informed say and impact on the decision-making process.

Within this complex field of governments' need for innovation and society's claim for more transparency and involvement the question is how Open Government can realize transparency, participation and collaboration in practice. Which formats, media and means of communication can help to realize this vision? OGD is seen as the first step in the realization of Open Government and as we will see in section 3 , a significant portion of OGD has a geospatial relation. This is to be expected - as we all live in space and our societies are spatially structured in many ways. We therefore propose space and time as possible integrators and facilitators for OGD and its use in the context of open governance. It can create an additional channel for citizens to engage, and maps and political participation make good partners (Crampton, 2009b).

\section{Space and Time as Integrator for OGD and Facilitator for Citizen Engagement}

With the rise of modern technology we encounter maps more frequently in our everyday lives, e.g. on our smartphones. Maps are tools that help us to navigate, explore, and understand the world we live in by providing context. In order to produce a map data is needed, particularly data that has a spatial relation to the subject at hand. Often this data is only valid for a certain period of time, and frequently, multiple dimensions are present and integrated on a map. Maps can easily answer four of the five W's in a visual way: what, when, and where. One might argue that the "who" can be answered as well depending on the case. Answering the "why" usually requires some reasoning of the map user or a specialized visualization. However, maps are often only used to depict data without means to query and use it. Frequently, they take the form of pre-produced 
images with a fixed meaning and geo-visualization (Elwood \& Leszczynski, 2013). This type of map lacks a key feature: interactivity, support for exploring, creating or editing a map itself.

Mapping data can be performative, participatory and political, while the map can serve as a comment and critique (Crampton, 2009b), or can show alternatives to the subject at hand. Maps are not simply images for people to look at. Behind every map is "a story to be read or a speech to be heard" (Sui \& Goodchild, 2003, p. 12). The speculation that Geographic Information Systems (GIS) will become a part of our media environment (Sui \& Goodchild, 2001), seems to hold true: They are not longer only used by experts to create maps or to perform spatial analyses. Online web mapping services and the rise of Volunteered Geographic Information (VGI) enabled a large portion of the population to create maps, and to share knowledge in the process (Sui \& Goodchild, 2011, p. 1738). The line between GIS and interactive maps has started to blur since both have become accessible online and are used to create, edit and explore spatial data.

Therefore, a shift of perspective is in order. Since everyone is inherently familiar with space and time, map-like visualizations can enable many people to engage with complex societal topics. By creating accessible (augmented) geo-visualizations, by making them intuitively interactive and by integrating open (government) data, we believe that citizens can be empowered to voice their wishes, comments and critiques more effectively, and that public engagement processes can be transformed.

With the rise of the Web 2.0 the mode of "only serving" information is challenged and has changed now. Lievrouw $(2011$, p. 15) states that "interactivity is a necessary cultural, social and technological condition that supports interaction, which in turn is a necessary condition for participation." She argues that it is a much smaller step from using or interacting to participating than from exposure or reception to participation. Research in the area of computer supported collaborative work (CSCW) supports this. It has been shown that social media can support political deliberations (Hemphill \& Roback, 2014; Semaan, Robertson, Douglas, \& Maruyama, 2014). Properly designed they can also help to provoke offline participation by bridging virtual and physical attendance (Huang, Wang, \& Yuan, 2014) or entice users to correct information while they consume it (Masli \& Terveen, 2014). In the case of Open Government, transparency and collaboration are two of the main goals. As such and in light of the participatory nature of Web 2.0 it makes sense to consider creating citizen engagement by enabling people to interactively explore OGD, to use it during communication and deliberation, and to contribute to OGD.

The argument being made here is in line with Crampton's (2001): Maps should be recognized as social constructs. While there are certainly cases where objective and quantifiable cartographic representations are essential (i.e. there is a need for a "finished" map), collaboration and participation are ongoing processes. Harley (1989) even argues that cartographic facts are only facts within a specific (cultural) perspective. Yet this is exactly what makes user-generated or edited maps based on OGD so valuable. They represent the view of the creator - may that be an individual or a CSO. They act as windows to their particular viewpoint, representing their needs and concerns. They can serve as condensation points for discussion if they are created openly and publicly in an iterative process. Users should thus be able to create maps on the spot as multiple competing visualizations de-emphasize the presentation in favor of the exploration of data (Crampton, 2001).

Participation and collaboration research related to GI Science has been conducted mostly in the field of Public Participatory GIS (PPGIS) or Participatory GIS (PGIS). P(P)GIS is a field of research focusing on strengthening the use of GIS to broaden public involvement in policymaking and promoting the value of GIS technology to non-expert groups like NGOs. Initially it focused on the ease of use of GIS technology to make spatial-decision making tools available for everyone involved in official decisions (Obermeyer, 1998; Sieber, 2006). Most of early research was technology-driven, rather than issue-driven (Dunn, 2007, p. 617) and has therefore been strongly criticized. 
Using accessible and interactive geo-visualizations as catalyst for citizen engagement inverts this approach since the emphasis is not on decision-making in the first place; rather, it is on engagement that may lead to participation and then collaboration. We understand maps as carrier of a message, as (part of) a canvas, like a spoken dialogue, a text or an image. This understanding is well reflected in Elwood's and Leszczynski's (2013) deliberations about how new spatial media artefacts and mediating technologies on the Internet - provide opportunities for civic activism and engagement to effect social change. This shift in perception that focuses on citizen engagement, knowledge production in participatory context and mapping is best reflected in the "Participatory Geoweb" research (Crampton, 2009a) - a term coined by Sieber in 2008.

The convergence of GIS and social media (Sui \& Goodchild, 2011) is therefore highly relevant as social media are perceived as just that - media to communicate. We aim to establish interactive geo-visualizations that integrate open (government) data in the same way, so that citizens can transport their wishes, comments or critiques on a medium that enables exploration and fosters reasoning in a visual and spatial way. We perceive those visualizations as an interactive medium and re-emphasize the role of speech, narrative and rhetoric as equally important to their visual or analytical components (Sui \& Goodchild, 2003; Sui, 2004).

The central challenges in realizing this approach are to find and process OGD, to conceptualize and create augmented interactive geo-visualizations as well as to integrate them into the citizen engagement process. Each challenge can be split into several interlinked tasks (see Figure 1) that we will briefly discuss here. Sections 5 and 6 will report in more detail on our ongoing work of the first two of those challenges: harvesting Open Government Data \& augmented interactive geovisualizations. We cannot yet report on the actual integration of the interactive geo-visualizations, but are cooperating with two CSOs to deploy them in the spring of next year in an actual participatory context.

Integrating OGD requires identifying relevant sources such as Open Government Portals (OGPs). In some cases one portal might federate all relevant data, while in others relevant OGD might be spread across several portals from different providers. Finding and filtering relevant OGD is thus an important step, usually facilitated by metadata and Application Programming Interfaces (APIs) or user interfaces built for this purpose. If the data is present in standard geospatial formats it can be processed directly, otherwise it has to be geo-referenced and transformed first. 
Augmented interactive geo-visualizations need to be designed in a user-friendly way. They are augmented as they are not only visualizing OGD but also offer additional information and functionalities that are strongly coupled to the geo-visualization. One example for this kind of augmentation is a chat functionality that allows for directly linking messages to geospatial features or geo-referenced textual descriptions, images and videos. The augmentations are complementing the interactive geo-visualization, helping citizen to contribute to the case. The means of interaction provided to interact with the geo-visualization and complementary visualizations play an important role as well. Complementary visualizations need to reflect the overall design so that a coherent user experience is achieved. It is crucial to consider the entire citizen engagement process as certain boundary conditions might apply and the geo-visualization needs to be integrated into the overall citizen engagement process.

The existing processes and states need to be analyzed and potentially adapted. The timeframe, target citizens and the intended type of the citizen engagement process (communication, consultation, participation, collaboration) are factors that can inform the design of the interactive geo-visualizations. In the following sections, we will discuss the three challenges depicted in Figure 1 in more detail. We will focus on the first two challenges and discuss the third one only briefly since work on the latter is still in an early stage while we can already report on experiences and results regarding the former.

\section{Open Data Harvesting \& Pre-Processing}

After the introduction and review of the different actors and concepts, this section reports on first hand experiences with geospatial OGD we collected. Geospatial Open Data is a fundamental element of our approach in terms of understanding and establishing augmented interactive geovisualizations as a tool to foster citizen engagement. Our report and reflections on OGD are based on OGD that is exposed through Germany's Open Data Portal (govdata.de) ${ }^{4}$.

OGD platforms cover aspects such as licensing, recency, data formats or basic categorizations. Visualizing, transforming or even remixing the data is beyond the scope of such portals, but they are nonetheless crucial if citizens are to engage in the governance process. In order to promote the usage of OGD, it is benefical to integrate it directly into the environments and workflows of potential

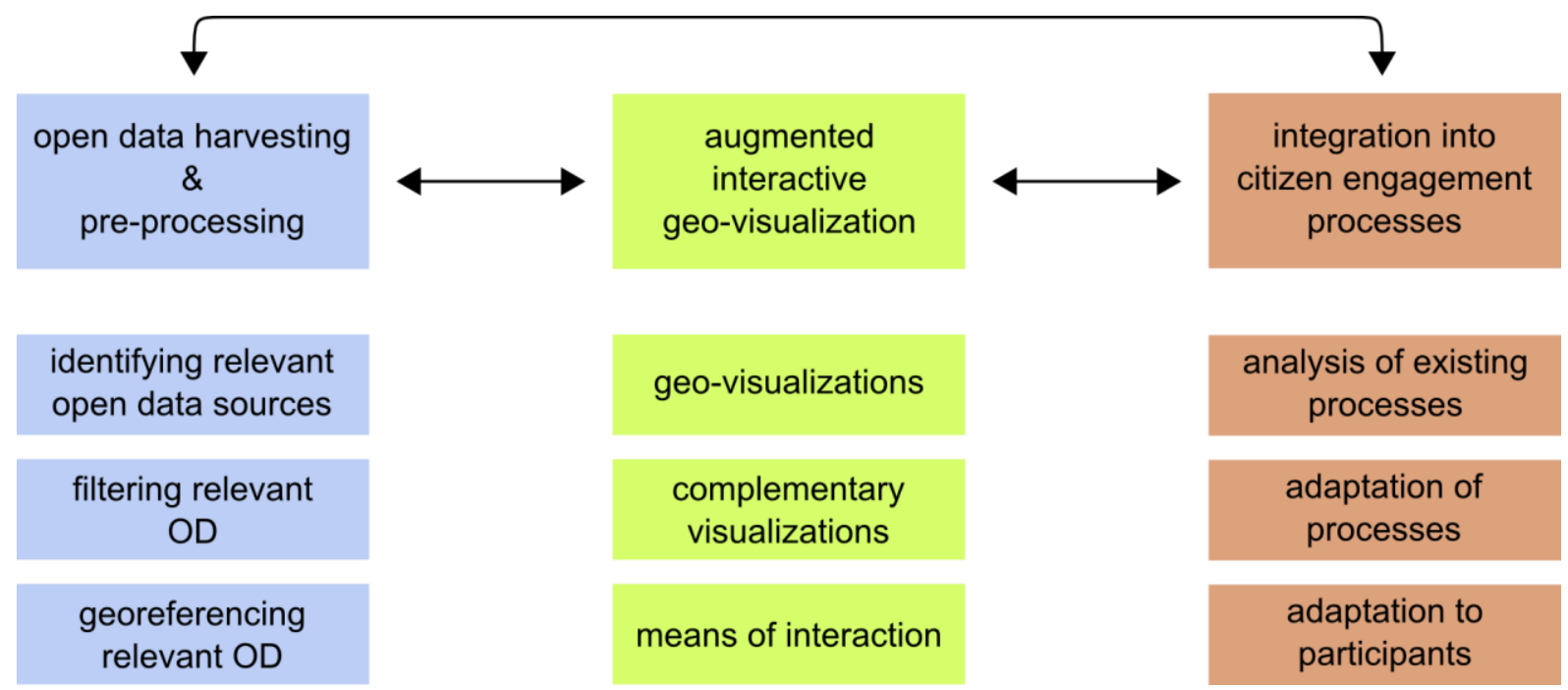

Figure 1: Three central challenges are present in order to realize and use augmented interactive geovisualizations. Each challenge consists out of interdependent steps that need to be taken. For a geovisualization Open (Government) Data needs to be harvested and pre-processed, the augmented interactive geo-visualization has to fit into the entire citizen engagement process that informs the means of interaction and possibly complementary visualizations. Furthermore the existing processes have to be adapted to the interactive geo-visualization, while the process and utilized geovisualization itself has to be adapted to the participants. 
users, which reduces the effort needed to use it. Typical workflows and processes are re-mixing and transforming OGD for actual use cases. For example, data journalists might create visualizations based on OGD. Local initiatives may use it to create flyers or posters for a certain topic and to rally citizens to support. Administrations, companies and CSOs could invite citizens to town-hall meetings discussing projects enriched and backed-up by OGD. All of these processes contextualize and transform OGD. As maps have reached public mainstream (Dunn, 2007, p. 618) and help in communication scenarios (Hopfer \& MacEachren, 2007) we have created a tool that exposes geospatail OGD directly within the work environments of map creators.

\section{Automatically Harvesting Geospatial Open Data}

To facilitate the use of geospatial OGD we cooperated with con terra and ESRI Germany to develop and deploy a software tool called "Open Data Bridge" that harvests govdata.de every week. The harvesting process is copying and transforming the metadata of the available OGD into the cloud-based infrastructure (ArcGIS Online) of our cooperation partner. It essentially creates a reference to the OGD within their infrastructure. Once this reference is available the OGD is seamlessly integrated and accessible into all of their products. The Open Data Bridge filters the available OGD on govdata.de and harvests datasets that are provided in standard geospatial formats through the API based on the Comprehensive Knowledge Archive Network (CKAN), which is offered by govdata.de. The cloud-based infrastructure of our cooperation partner offers a public API to enable external use, which we rely on as well. In its current form the Open Data Bridge harvests Web Mapping Services (WMS), Keyhole Markup Language (KML) and Comma Separated Value (CSV) files. The former two (WMS \& KML) are standard approved by the Open Geospatial Consortium $(\mathrm{OGC})^{5}$, while the latter is a lightweight standard for exchanging tabular data. The Open Data Bridge is freely available on GitHub as open-source under an Apache 2.0 license ${ }^{6}$.

At the time of writing govdata.de featured 8017 unique datasets that were exposed through the CKAN API. In total 4879 of these datasets were harvested by our software. The large majority of the harvested datasets (4829) was available as WMS while the remaining harvested datasets were KML or CSV files. The reported numbers vary since the number of datasets provided through govdata.de changes due to datasets being updated or removed. Additionally, we are not harvesting every single dataset provided by govdata.de. Datasets can be offered in multiple formats (called resources) in govdata.de, and the Open Data Bridge currently prioritizes WMS files over KML and CSV as WMS facilitates the integration of datasets with the available tools most readily. While we cannot present reliable access numbers to the harvested resources, our own incomplete tracking suggests the datasets are currently not accessed by large numbers of interested parties. Only a few of them were used a couple of hundred times - see section 7 for potential reasons.

While WMS and KML are both formats that are inherently spatial and can reliably be mapped, CSV files contain only tabular data that are not inherently spatial. In the latter case, metadata fields can indicate a geospatial component but they are optional. Therefore, they cannot reliably be used within govdata.de's metadata schema and can only serve as indicators.

\section{Identifying Open Data with a Geospatial Component}

Since this issue of incomplete, missing or incorrect metadata is a general one, we developed a data-mining tool to mitigate it. This software uses decision trees (Safavian \& Landgrebe, 1991) to identify geospatial information in CSV files based on their actual content and metadata, and according to their spatial granularity. The CSV data mining software uses a dual approach classifying the present metadata in govdata.de and the actual data of the CSV files separately for each dataset. The reason is simple: A metadata classification is not inevitably "correct" for the

\footnotetext{
${ }^{5}$ http://www.opengeospatial.org/standards

${ }^{6}$ https://github.com/EsriDE/OpenDataBridge
} 
actual dataset. Mistakes may have happened at the time of publishing the metadata, it may simply be incomplete, or it might have been created without considering the importance of good metadata. To avoid incorrect classifications due to this fact, the CSV data mining tool maintains two separate classifications for each dataset. By comparing the two different classifications (metadata-based and based on the actual file content), it is possible to check for potential errors in both classification approaches. This comparison process also facilitates the identification of the most common mistakes that the authors publishing the datasets made.

We developed a custom decision tree for the metadata-based classification as many relevant fields were too varied or were not present at all, which ruled out a standard classification algorithm.

Three decision trees were generated for the actual data classification of the CSV files based on the well-studied algorithms ID3 (Quinlan, 1986), C4.5 (Quinlan, 1996) and Cart (Breiman, Friedman, Stone, \& Olshen, 1984). The results of the three data-based classification decision trees were compared to investigate which of them provided the best results. All three decision trees classified each dataset into the same categories - which indicates a representative training set, but happened most likely due to the fact that the CSV datasets are all fairly homogenous resulting in a small amount of edge cases. The two most prolific publishers of CSV files in govdata.de are the "Federal Statistical Office" (1313 entries) and the "Statistical Offices of the Federation and the Federal States" (819 entries), while the remaining entries are provided by several smaller publishers.

Our results of the automatic CSV file classifications were tested against a manually categorized set. This manually created sample categorization was based on $10 \%(224)$ of the available datasets equally distributed across the publishers. The different categories for the classification of the datasets are straightforward and extend the suggested categories of govdata.de's metadata. We classified the datasets into the following spatially relevant categories: federal republic, federal state, district, city, address, point and no classification.

Table 1: Results of the classification of the CSV data mining software for the metadata and the actual data in the CSV files. The percentages are rounded. The data-based and metadata-based classifications overlap largely. $87 \%$ of classified datasets are in the same classes in both processes.

\begin{tabular}{|l|r|r|r|r|}
\hline spatial relation & \multicolumn{2}{|c|}{ data-based classification } & \multicolumn{2}{c|}{ metadata-based classification } \\
\hline & \multicolumn{1}{c|}{$\%$} & number of files & \multicolumn{1}{c|}{ \% } & \multicolumn{1}{c|}{ number of files } \\
\hline federal republic & 45.1 & 1013 & 42.6 & 287 \\
\hline federal state & 10.9 & 245 & 12.7 & 745 \\
\hline district & 32.4 & 728 & 33.2 & 93 \\
\hline city & 1.4 & 31 & 4.1 & 23 \\
\hline address & 1.9 & 42 & 1.0 & 5 \\
\hline point & 3.0 & 68 & 0.2 & 137 \\
\hline no classification & 5.3 & 118 & 6.1 & \\
\hline
\end{tabular}

For the data-based classification we found that $1.4 \%$ of our categorizations were incorrect. Most of the erroneous classifications happened due to ambiguous keywords. For example, "Germany" can indicate a spatial relation but could also be part of a political party's name, e.g. "Social Democratic Party of Germany". If other stronger indicators for a spatial relation were found, this aspect did not result in an erroneous classification. 3.2\% of the metadata-based classifications were incorrect. As with the data-based classification this happened due to keywords within the metadata that have an ambiguous meaning and thus resulted in a wrong indication of a spatial relation in our geo-referencing process. $87 \%$ of the datasets are in the same classes for both approaches. While a more thorough analysis is underway we noted a trend in the data: as the spatial granularity increases the quality of the metadata decreases. Often the metadata does not 
indicate if the dataset includes addresses or point data. Instead it features the rough localization with the next lower spatial granularity. See Table 1 for the results of both classifications.

Furthermore, we observed that some CSV files did not follow the convention that the first row should contain metadata about the following rows. Sometimes, the first row already contains data. On other occasions, the first few rows contained comments on the following data and / or referenced external sources. In some cases, publishers provided metadata and explanations in different resources of the same dataset: e.g. the CSV file contains the data itself and a PDF resource featured metadata for that dataset. Such inconsistencies are likely to confuse OGD users who expect that each resource is self-contained, e.g. the datasets provide all information needed to interpret the data provided. Those kinds of errors will hopefully vanish as publishers become more familiar with the publishing process, the guidelines and basic conventions.

In summary, we have developed at tool that can automatically harvest and filter geospatial OGD so that it can be directly integrated into the workflows and tools of map creators. The software is publicly available, and can thus be extended or adapted by any interested party. The tool relies on decision trees to classify and categorize arbitrary data that is stored in CSV-files based on their spatial granularity and relation. This process can be used to complement existing metadata that is often lacking or does not expose relevant information properly. We are currently investigating if this process can be successfully applied to other data formats as well. The data mining software is available as open-source software under an MIT license to allow other researchers to adapt or enhance it for their purposes ${ }^{7}$.

\section{Using Space and Time in Citizen Engagement}

The previous section looked at the first challenge of our approach, and it elaborated on how OGD with geospatial components can be harvested, exposed and identified. This section takes a look at the second challenge: we review three approaches of interactive geo-visualizations that could facilitate citizen engagement in the Open Government context. The first tool changes the way we work together with maps. It introduces the notion of synchronous, distributed, real-time online mapping as one mode of operation for OGls (section 4.1). Maps as spatial dialogue platforms are reviewed in section 4.2. We discuss why maps can be useful in public online dialog platforms when they are treated as "first class" citizen and designed to support an exchange of textual and visual representations equally. Lastly, we propose the use of space and time to highlight engagement opportunities through Location Based Services (LBS) in section 4.3.

\section{Synchronous Distributed Online Collaborative Mapping}

We understand maps as an inherently interactive medium, and it is thus important to investigate means and modes of interaction and how they affect participation and collaboration in multi-user scenarios. Usually editing or mapping data is an asynchronous and blocking operation performed by an individual. Others can only work on the data sequentially or risk creating inconsistent versions. If they are interested in map manipulation as they occur, they have to be at the same place or use remote presence systems to "observe" the current mapper. Even then, they are still not able to actively manipulate the map. They are not "drivers" but merely "passengers". This issue can be addressed by enabling users to collaborate in real-time and online rather than locally editing documents, which then have to be shared and merged manually.

This notion of synchronous (distributed) real-time collaborative mapping is inspired by popular real-time collaborative web editors such as Google Drive, Microsoft Live, Cloud9 IDE or Etherpad ${ }^{8}$.

\footnotetext{
${ }^{7}$ https://github.com/ReneUnrau/GovData-Parser

${ }^{8}$ http://drive.google.com, http://onedrive.live.com, http://c9.io, http://etherpad.org accessed 27 July 2014
} 
Our real-time collaborative mapping platform is called Ethermap (see Figure 2 for an image of the application). It realizes functionalities of collaborative text editing on maps for the first time.

In this context, real-time collaboration refers to the concept of allowing users to simultaneously edit the same dataset while working on different devices and at different places. When users are working in the same editor, any changes are immediately visible to all collaborators (Koren, Guth, \& Klamma, 2013). It has been shown that this functionality can increase efficiency for text-based editors compared to standard approaches (Goldman, Little, \& Miller, 2011). In addition to an increased efficiency, every connected user can directly interact with every element of a map at the same time. This is a qualitatively different experience than just "witnessing" an edit or the creation of map elements, and it aligns well with the participatory nature of OGls. If users want to contribute or collaborate, they should be able to do so immediately without waiting for someone else to finish. This synchronous, distributed, real-time interaction also challenges the very idea of what defines a map user (Crampton, 2001) since every connected user can easily become a contributor through seamless drop in and drop out mechanisms.

Collaborative real-time work environments and interactions have a number of key requirements in order to work properly. User awareness and version control are particularly crucial as users have to know where others are currently working to avoid conflicts. They should also be able to distinguish between different users and communicate with each other (Antunes, Herskovic, Ochoa, \& Pino, 2014; Heinrich, Lehmann, Springer, \& Gaedke, 2012). Version control systems enable users to review changes over time, and they are beneficial for understanding decisions or for reverting to a specific revision (Grishchenko, 2010). Furthermore, version control systems show who edited a specific part, which helps to evaluate authenticity. In Ethermap, we have realized key features related to user awareness such as color-coding users; a chat that can refer to spatial objects; or highlighting and following work areas of other users as well as indicating currently edited features. We also implemented standard version control mechanisms for geospatial vector data. The version control mechanism additionally includes a "playback" function that can be used to go through the creation process step by step. This function can help users to understand how a certain decision was made and what alternatives were considered during the process. A history function is available as well: it provides a graphical interface for the inspection for each feature on the map and enables users to revert back to a previous version of that feature if necessary. 


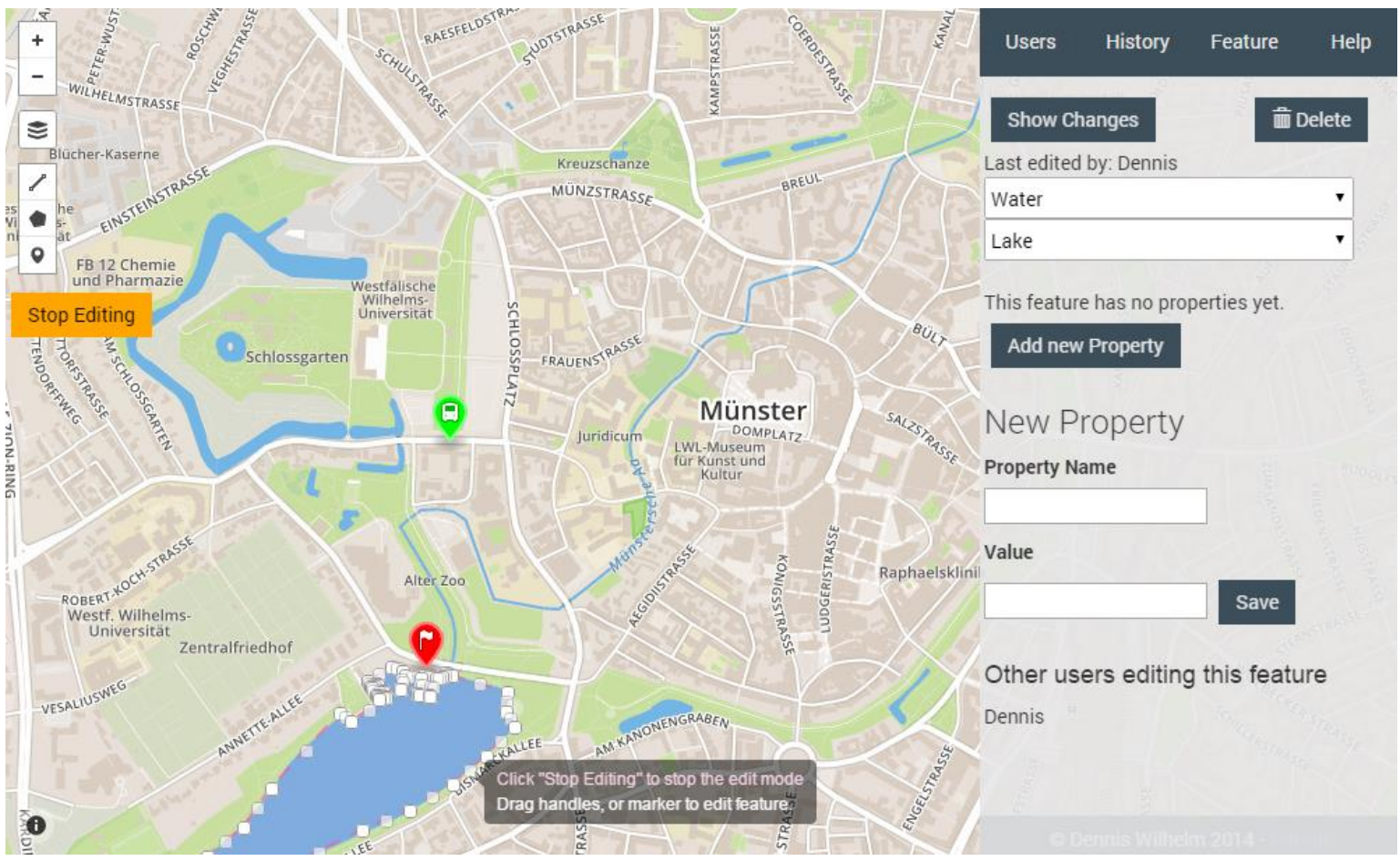

Figure 2: Ethermap - the map shows a polygon currently being edited (indicated by the dashed line), and a context-sensitive menu on the right displays the attributes of this geo-object. The polygon is currently being edited by another user. It is possible to help the other users editing the same geo-object directly or by working on any other part of the map, which enables actual real-time collaboration. The entire application can be accessed through any modern web-browser and thus keeps the usage and entry barrier low.

Ethermap is based on a client server architecture built on top of current web technologies and open source frameworks such as WebSockets, HTML5, Node.js and a No-SQL database. It is available as open-source software under an Apache 2.0 license $^{9}$. We are currently conducting expert interviews and a large-scale user-study to test the usability and feasibility of our approach. It is the first mapping platform of its kind that allows distributed synchronous real-time mapping enabling actual collaboration. Users can work together ad-hoc and simultaneously on a map without being relegated to being "backseat drivers". Furthermore, it seamlessly supports asynchronous work modes and is thus well suited for OGls that often rely on common workspaces in (a)synchronous work time with ad-hoc networking opportunities (Geiger \& Lucke, 2012).

\section{Maps as Spatial Dialog Platforms}

Online public consultation or collaboration software allows citizens to voice their opinions or concerns while arguments or discussion are exchanged textually. Such systems often neglect the spatial dimension except for "pinning" arguments to a place - a concept developed more than a decade ago (Rinner, 1999). While geo-visualizations are usually present somewhere in such systems, they are not treated as "first class" citizens.

MacEachren et al. (2005) showed the potential of map-mediated dialogs between human collaborators and went on to examine how geospatial annotations support collaboration from a communication theory viewpoint (Hopfer \& MacEachren, 2007). They argue that geospatial annotations accelerate insights in group discussions into what is known, who knows what, how a

\footnotetext{
${ }^{9}$ https://github.com/dwilhelm89/Ethermap
} 


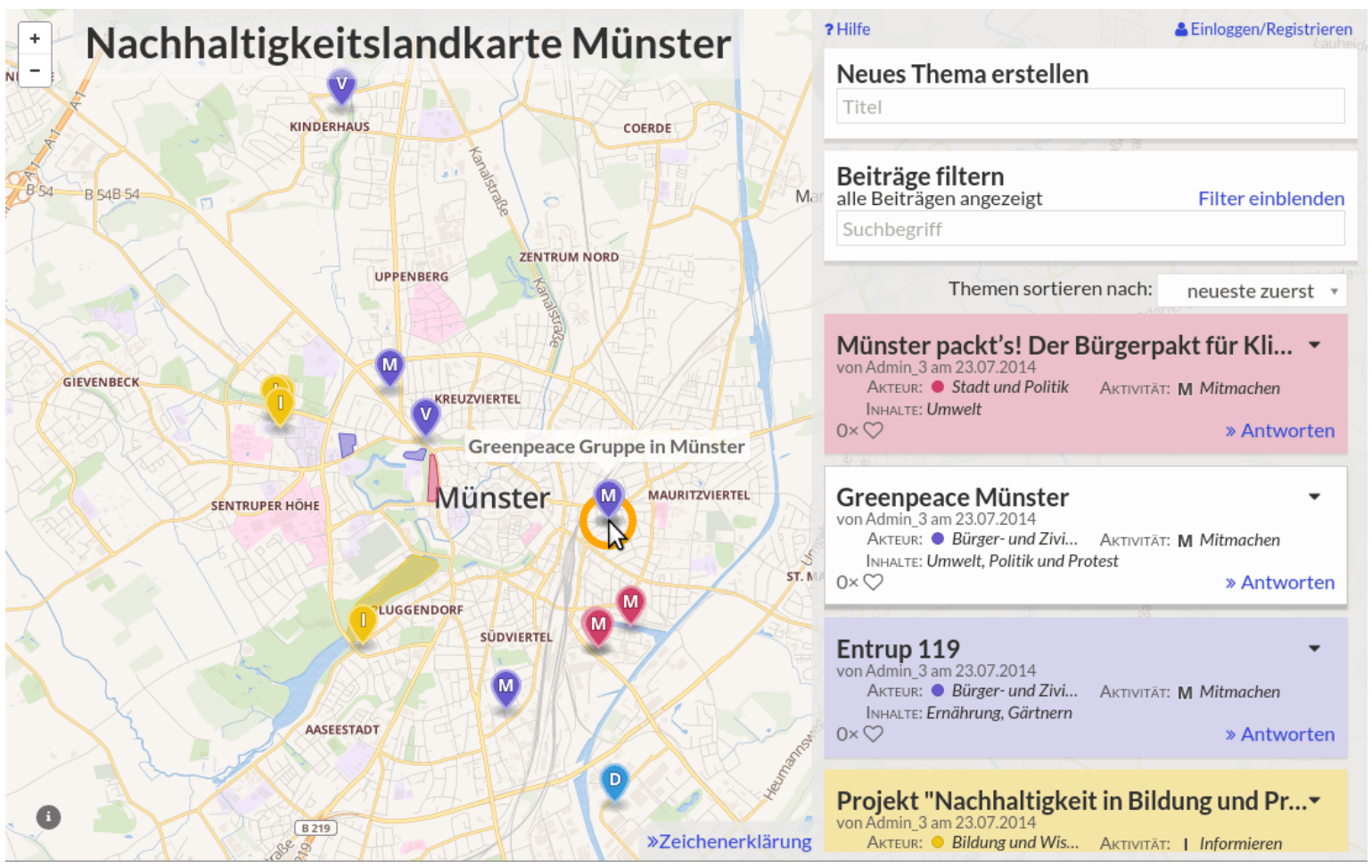

Figure 3: A "Dialog Map" that displays engagement opportunities for sustainability projects and open issues. An engagement opportunity (Greenpeace Münster) is currently highlighted by a user. Users can drill down into specific topics, search them visually on the map, and use a textual search or filters. Users can also discuss and contribute text as well as locations to each topic, and they can also create new topics.

problem is understood by group members, and how a problem is negotiated by group members over time. Additionally, they argued that maps can actively encourage explicit contributions of knowledge, reduce redundant information and highlight underrepresented views.

While several iterations of the idea of maps as argumentation platform have been proposed (Kessler, Rinner, \& Raubal, 2005; Rinner \& Bird, 2009; Rinner, 2005, 2006, 2007), they are limited in a number of ways. In particular, they suffer from complex user interfaces, limited spatial grounding, split views for discussions and the map, awkward textual representations or an explicit focus on urban planning scenarios. Therefore, we are currently re-iterating the design and concept in cooperation with two CSO's in an agile process. We place an emphasis on engaging citizens in discussions through spatially exploring them. The user-interface was designed and iterated over several times and was strongly influenced by the feedback from the CSOs. This visual experience can help citizens to generate knowledge through interactively exploring the geo-visual artifacts (Elwood \& Leszczynski, 2013). The goal is to develop a dialog map (see Figure 3), with the map as "first class" citizen - acting as a "spatial window" to find dialogs that are meaningful to the citizen through their spatial relation. User contributions or generated content in online systems has often a strong "local" relation - meaning that they actively engage if there is a relation to where they live (Hecht \& Gergle, 2010). Directly exposing and expressing this "localness" in an interactive, visual and exploratory way could help to engage citizens in discussions.

Previous iterations of argumentation maps did not treat maps in this way: the map was often just another mode of finding threaded discussions, thereby undermining the potential of geospatial annotations. Our approach however is inherently spatial: the map structures the discussion and all discussions are related and grounded in space. Therefore, dialogs can be found through the map, which acts as interface, and every part of a dialog can be referenced (spatially) in a " $n$ to m" way. 
The interface is contextually enabled: it simultaneously highlights dialogs and arguments in the textual representation as well as on the map when a user is engaging with them. It also only shows related and relevant information, which reduces information clutter. In addition, we facilitate the reuse of arguments via linking to them as this has been shown to help reflecting one's thoughts (Kriplean, Morgan, Freelon, Borning, \& Bennett, 2012). Additionally, we investigate how to best support arguments with factual data, e.g. by offering users the ability to attach or link to further sources (such as OGD in particular). Authoritative data could help to improve discussions in a way as similar to fact checking mechanisms provided by a trusted official third party in an online public dialog (Kriplean, Bonnar, Borning, Kinney, \& Gill, 2014). The first prototype of our spatial discussion platform is currently being evaluated with two CSOs in real-world scenarios. Following the approach used to realize Ethermap, the spatial discussion application also relies on modern Web technologies. It is available as open-source under an Apache 2.0 license $^{10}$ to allow other researchers and practitioners to enhance or modify the application for their own scenarios.

\section{Highlighting Engagement Opportunities Spatially and Temporally}

Our final example linked to the second challenge (see Figure 1) relates to communicating and highlighting engagement opportunities to citizens. Information about such opportunities is usually communicated in different modes, push or pull-based, and through different channels. Traditional channels in the form of direct mailings, advertisements, flyers or info boxes have recently been complemented by social media. Social media perpetuates personal sharing through recommendations in a "word of mouth" process. Though the "when" and "where" are essential aspects for every engagement opportunity they are currently are not communicated very effectively by existing means of promoting such opportunities.

The rise of ubiquitous computing and social media offers a promising opportunity to address this issue in the form of Location Based Services (LBS) that enhance the user-experience by integrating the user's current location, context and activity. Instead of only communicating to a citizen through traditional or social media channels when, where and how engagement is possible, space and time could be used more effectively via geofencing. The idea of geofencing or locationbased notifications itself is not new (Munson \& Gupta, 2002) and is used in various forms for marketing, advertisements or vehicle tracking. So far, it has not been used much in citizen engagement contexts. A geofence is a geographic area that is used to trigger and send notifications or messages to subscribers when they enter or leave an area. This can be paired with time-based constraints (i.e. only alert people when they enter or leave at certain times). Locationbased notifications can thus serve as location-based reminders, which only show up if they are useful in the current context (Sohn et al., 2005).

This general approach can be used in citizen engagement scenarios in various ways: Organizers can create geofences around places that host regular activities or that are affected by some development. This might be a town hall where meetings take place or a district, street or plaza that is the subject of the citizen engagement. Messages can then be pushed to citizens that have a "City" or "Citizen" app on their smartphone as they enter or leave the corresponding areas. This in turn can create a stronger connection as the person is in the immediate vicinity of the place in question at the time when they receive the notification (Han, Shih, Rosson, \& Carroll, 2014). In addition to being used as a push-based communication triggered by place and time, geofences can also act as spatial filters that are created by citizens. Notifications are sent when in an area they specified an opportunity for engagement opens up, or when it is affected by some development. Similarly, location-based reminders could help citizens to remember certain aspects of engagement opportunities, e.g. a poll or consultation that is soon taking place or to rally for support at another

\footnotetext{
${ }^{10} \mathrm{https}$ ://github.com/ubergesundheit/dialogmap
} 
event. These spatial reminders could also be shared and thus lead to opportunistic, location- and crowd-based mobilization.

We are currently working on an overall communication concept that uses geofences and location-based messages to highlight engagement opportunities. Our investigations focus on the design of a "citizen" app providing such services, and on how to best integrate the creation of the messages and geofences into existing structures and processes.

\section{Discussion}

In this article we propose to use augmented interactive geo-visualizations integrating Open Data sources to enable people to engage with complex societal topics in Open Government initiatives. Our approach outlines the necessary steps, and we have reported on initial work towards addressing two of the three major challenges we identified. Compared to traditional analogue and digital means to realize citizen engagement, the proposed approach promises to offer a number of benefits. Firstly, it can enable participants to access and use OGD effectively by using space and time as integrators, e.g. to support their argument, to assess the feasibility of their suggestions, and to analyze proposals put forward by others. We envision that this aspect can lead to more informed discussions, and we also see potential for people feeling empowered and consequently participating more frequently. Secondly, using interactive geo-visualizations can make complex issues more accessible to a broader audience as it builds on the common concepts of space and time, e.g. in the form of maps. These visualizations can also present discussions in a different way that avoids issues often observed with sequential (text-based) modes of presentation such as the repeated presentation of the same argument or the overlooking of alternatives presented earlier due to people only reading / seeing the last few messages in a thread. In addition, filtering by space and time allows citizens to focus more easily on specific aspects that they are interested in. One of the biggest benefits of visualizing (geospatial) data is the possibility to visually search it, enabling people to process large amounts of data in parallel, e.g. to quickly find anomalies or patterns. Additionally, visualizations extend our memory from the way a symbol or image evokes non-visual information (Ware, 2014, p. 145 \& p. 352). However, despite these benefits compared to textual forms, visualization can lead to the exclusion of certain groups, e.g. to impairments such as (color-) blindness.

The proposed approach is also subject to some further limitations. Compared to traditional approaches, it may require more sophisticated hardware and may be unfamiliar initially. Some cases of citizen engagement might not have a strong spatial and / or temporal component, e.g. when issues such as tax laws are discussed. In such cases, our approach might be less useful. However, in many common cases where citizen engagement is asked for, space and time play a key role, e.g. in urban planning. In the following paragraphs we discuss the three central areas of our approach and the corresponding implications and limitations in more detail.

In section 3 we reported on our experiences in harvesting, filtering and providing OGD that has a geospatial component. One of the most crucial observation was that most of the available OGD was published in a format that does not adhere to the Open Data spirit. WMS services distribute their data as image tiles - as raster data, even if the source data is vector based. This prohibits the easy downloading and modifying of the data (Johnson \& Sieber, 2012). Therefore, we are currently investigating how to incorporate more vector-based formats, e.g. by integrating Web Feature Services (WFS) or the community standard GeoJSON. Our first experiences indicate that vector data that is curated and published as Open Data by our cooperation partner received thousands instead of hundreds of hits. However, if the provided OGD is mundane, meaningless or the formats are not really "open" there is the risk of a data provider just pretending to be open when it is actually not (Yu \& Robinson, 2012).

Metadata accompanying OGD is crucial as well. In the process of identifying the geo-spatial relation in CSV-files we assumed that optional metadata fields such as "spatial-reference" or "bounding-box" would still be of help for the classification. It became clear during the development 
that if they are used at all they often contain indicators that are too broad. The "bounding-box" fields for example often refer to Germany as a whole, while the "spatial-reference" field does not contain the geographic coverage in a standard nomenclature but instead an arbitrary word. Nevertheless, our experiences with govdata.de indicate that the metadata frequently describes the data stored in the CSV files well. Although the user has to infer the content and spatial relation from required metadata field such as the "title", the "author" or the textual description of the dataset. This complicates the automatic processing and further use of Geospatial Open Data as specialized software and algorithms (such as decision trees) are required to "infer" the content type. We are currently investigating if this approach is feasible for other resource types and formats as well.

Although it might complicate the publishing process of OGD we think that certain metadata fields should be mandatory or at least suggested dynamically based on the resource types that are published. To give an example: If a GeoJSON or WMS resource is present alongside a CSV resource, it is straightforward to expect that the dataset contains points, lines or polygons and to require the corresponding metadata fields. More advanced portals might even be capable of filling out metadata fields automatically and present them for a review by the publisher as certain resources of the dataset already contain metadata (e.g. the capabilities document of a WMS).

The second challenge we reported on is the design and use of interactive geo-visualizations. We proposed three distinct approaches that are currently being evaluated, but can already report on general implications and limitations. Map literacy and education are important in the light of such participatory systems (Crampton, 2009b) as map and GIS functionality can be misused or misinterpreted. Almost all of the web-based maps are biased as they heavily distort sizes and assume a spherical earth, which leads to misinterpreted relations. This is a general problem with all map-like visualizations as every map unfolds a three-dimensional object to a two dimensional plane. There is no "correct" way to project maps, only approximations that work well for a certain case. The geo-visualizations that are produced or used in citizen engagement cases represent one possible view that is influenced by the specific perception of the discussion partners. This makes them valuable, but everyone involved has to be aware of it. They should not be evaluated by the same scientific and cartographic standards such as objectivity, accuracy or truthfulness (Harley, 1989).

We cannot yet provide substantial insights into the integration into citizen engagement cases as we are only now preparing for an actual deployment of the proposed concept with two CSOs. While we hope to obtain insights into the interaction between the described tools and the engagement process, only long-term studies will show the potential of geo-visualizations in the Open Government context and how the process of engagement changes in response.

\section{Conclusion}

In this paper we introduced an approach that uses the universally familiar dimensions of space and time as common integrators to facilitate citizen engagement. Based on these dimensions, we proposed to integrate increasingly available Open Data sources with augmented interactive geovisualizations to create accessible tools that support citizen engagement. We identified key challenges as well as several essential advantages of this approach such as empowering people to support their arguments with data or avoiding issues often observed in sequential media (i.e. purely textual presentations). In order to demonstrate the feasibility of the proposed approach, we report on a number of software components and concepts as well as our initial experiences with them. This analysis looked at a tool to harvest and analyze Open Data sources to link them to spatiotemporal concepts as well as at different ways to use map-based visualizations in the context of citizen engagement.

The initial experiences and results we obtained indicate that our approach is not only technically feasible but that it also has the potential to empower citizens to more effectively engage with societal and governmental processes. While the approach is not equally feasible for every type of citizen engagement, in many cases there is a spatial and / or temporal dimension, which can then 
be exploited by the proposed approach. Nevertheless, more research is needed to fully develop, deploy and evaluate the approach. We are currently finishing two tools to support collaborative mapping and map-based discussion. In addition, we are designing a third tool aimed at highlighting engagement opportunities in situ. Now that initial tests on them have been completed, the next steps will be to deploy them in real world scenarios, i.e. in actual processes where citizen engagement is desired. Our article also reports on several implications and limitations of the approach e.g. the need for map literacy or challenges in harvesting OGD. Nonetheless we are confident that interactive geo-visualization can act as a catalyst for citizen engagement.

\section{References}

Almond, G. A., \& Verba, S. (1963). The Civic Culture: Political Attitudes and Democracy in Five Nations.

Antunes, P., Herskovic, V., Ochoa, S. F., \& Pino, J. A. (2014). Reviewing the quality of awareness support in collaborative applications. Journal of Systems and Software, 89, 146-169. doi:10.1016/j.jss.2013.11.1078

Ariely, G. (2013). Public administration and citizen satisfaction with democracy: cross-national evidence. International Review of Administrative Sciences, 79(4), 747-766. doi:10.1177/0020852313501432

Arnstein, S. R. (1969). A Ladder Of Citizen Participation. Journal of the American Institute of Planners, 35(4), $216-224$. doi:10.1080/01944366908977225

Barnickel, N., \& Klessmann, J. (2012). Open Data - Am Beispiel von Informationen des öffentlichen Sektors. In U. Herb (Ed.), Open Initiatives: Offenheit in der digitalen Welt und Wissenschaft (pp. 127-157). Saarbrücken: Universaar Saarland University Press.

Berman, S. (1997). Civil Society and the Collapse of the Weimar Republic. World Politics, 49(3), 401-429.

Bob, C. (2012). Civil and Uncivil Society. In M. Edwards (Ed.), The Oxford Handbook of Civil Society (pp. 209-219). Oxford et al.: Oxford University Press. Retrieved from http://www.oxfordhandbooks.com/view/10.1093/oxfordhb/9780195398571.001.0001/oxfordhb-9780195398571

Breiman, L., Friedman, J., Stone, C. J., \& Olshen, R. A. (1984). Classification and Regression Trees (1st ed.). Chapman \& Hall/CRC.

Carpini, M., Cook, F., \& Jacobs, L. (2004). Public deliberation, discursive participation, and citizen engagement: A review of the empirical literature. Annu. Rev. Polit. Sci. Retrieved from http://www.annualreviews.org/doi/abs/10.1146/annurev.polisci.7.121003.091630

Chambers, S., \& Kopstein, J. (2001). Bad Civil Society. Political Theory, 29(6), 837-865. Retrieved from http://www.jstor.org/stable/3072607

Cohen, J. L., \& Arato, A. (1994). Civil society and political theory. Massachusetts: MIT Press.

Cornwall, A., \& Gaventa, J. (2000). From Users and Choosers to Makers and Shapers Repositioning Participation in Social Policy. IDS Bulletin, 31(4), 50-62. doi:10.1111/j.1759-5436.2000.mp31004006.x

Crampton, J. W. (2001). Maps as social constructions: power, communication and visualization. Progress in Human Geography, 25(2), 235-252. doi:10.1191/030913201678580494

Crampton, J. W. (2009a). Cartography: maps 2.0. Progress in Human Geography, 33(1), 91-100. doi:10.1177/0309132508094074

Crampton, J. W. (2009b). Cartography: performative, participatory, political. Progress in Human Geography, 33(6), 840848. doi:10.1177/0309132508105000

De Tocqueville, A. (1985). Über Die Demokratie in Amerika. Stuttgart: Reclam.

Dunn, C. E. (2007). Participatory GIS a people's GIS? Progress in Human Geography, 31(5), 616-637. doi:10.1177/0309132507081493 
Eaves, D. (2010). After the Collapse: Open Government and the Future of Civil Service. In D. Lathrop \& L. Ruma (Eds.), Open Government: Collaboration, Transparency, and Participation in Practice (pp. 139-152). Beijing et al.: O'Reilly. Retrieved from http://oreilly.com/catalog/errata.csp?isbn=9780596804350

Eikenberry, A. M. (2007). Nonprofit Organizations, Philantrophy, and Democracy in the United States. In R. C. Box (Ed.), Democracy and Public Administration (pp. 169-194). New York et al.: M.E.Sharpe.

Elwood, S., \& Leszczynski, A. (2013). New spatial media, new knowledge politics. Transactions of the Institute of British Geographers, 38(4), 544-559. doi:10.1111/j.1475-5661.2012.00543.x

European Parliament. (2011). Open data An engine for innovation, growth and transparent governance (pp. 1-13). Retrieved from http://eur-lex.europa.eu/LexUriServ/LexUriServ.do?uri=COM:2011:0882:FIN:EN:PDF

Gaventa, J. (2002). Exploring Citizenship, Participation and Accountability. IDS Bulletin, 33(2), 1-14. doi:10.1111/j.17595436.2002.tb00020.x

Geiger, C. P., \& Lucke, J. Von. (2012). Open Government and ( Linked ) ( Open ) ( Government ) ( Data ). eJournal of eDemocracy \& Open Government, 4(2), 265-278.

Goldman, M., Little, G., \& Miller, R. C. (2011). Real-time collaborative coding in a web IDE. In Proceedings of the 24th annual ACM symposium on User interface software and technology - UIST'11 (p. 155). New York, New York, USA: ACM Press. doi:10.1145/2047196.2047215

Goodsell, C. T. (2006). A New Vision for Public Administration. Public Administration Review, 66(4), 623-635. doi:10.1111/j.1540-6210.2006.00622.x

Grishchenko, V. (2010). Deep hypertext with embedded revision control implemented in regular expressions. In Proceedings of the 6th International Symposium on Wikis and Open Collaboration - WikiSym '10. New York, New York, USA: ACM Press. doi:10.1145/1832772.1832777

Hamilton, M. R. (2007). Democracy and Public Service. In R. C. Box (Ed.), Democracy and Public Administration (pp. 3-20). New York et al.: M.E.Sharpe.

Han, K., Shih, P. C., Rosson, M. B., \& Carroll, J. M. (2014). Enhancing community awareness of and participation in local heritage with a mobile application. In Proceedings of the 17th ACM conference on Computer supported cooperative work \& social computing - CSCW'14 (pp. 1144-1155). New York, New York, USA: ACM Press. doi:10.1145/2531602.2531640

Harley, J. B. (1989). Deconstructing the map. Cartographica: The International Journal for Geographic Information and Geovisualization, 26(2), 1-20. Retrieved from http://utpjournals.metapress.com/index/e635782717579t53.pdf

Hecht, B. J., \& Gergle, D. (2010). On the "localness" of user-generated content. In Proceedings of the 2010 ACM conference on Computer supported cooperative work - CSCW'10 (p. 229). New York, New York, USA: ACM Press. doi:10.1145/1718918.1718962

Heinrich, M., Lehmann, F., Springer, T., \& Gaedke, M. (2012). Exploiting single-user web applications for shared editing. In Proceedings of the 21st international conference on World Wide Web - WWW '12 (p. 1057). New York, New York, USA: ACM Press. doi:10.1145/2187836.2187978

Hemphill, L., \& Roback, A. J. (2014). Tweet acts. In Proceedings of the 17th ACM conference on Computer supported cooperative work \& social computing - CSCW '14 (pp. 1200-1210). New York, New York, USA: ACM Press. doi:10.1145/2531602.2531735

Hopfer, S., \& MacEachren, A. M. (2007). Leveraging the potential of geospatial annotations for collaboration: a communication theory perspective. International Journal of Geographical Information Science, 21(8), 921-934. doi:10.1080/13658810701377780

Huang, A.-J., Wang, H.-C., \& Yuan, C. W. (2014). De-virtualizing social events. In Proceedings of the 17th ACM conference on Computer supported cooperative work \& social computing - CSCW'14 (pp. 436-448). New York, New York, USA: ACM Press. doi:10.1145/2531602.2531606 
Johnson, P. A., \& Sieber, R. (2012). Motivations driving government adoption of the Geoweb. GeoJournal, 77(5), 667-680. doi:10.1007/s10708-011-9416-8

Jonas, J., \& Harper, J. (2010). Open Government: The Privacy Imperative. In D. Lathrop \& L. Ruma (Eds.), Open Government: Collaboration, Transparency, and Participation in Practice (pp. 321-331). Beijing et al.: O'Reilly.

Kessler, C., Rinner, C., \& Raubal, M. (2005). An argumentation map prototype to support decision-making in spatial planning. In Proceedings of AGILE (Vol. 5, pp. 26-28).

Klessmann, J., Denker, P., Schieferdecker, I., \& Schulz, S. E. (2012). Open Government Data Deutschland. Eine Studie zu Open Government in Deutschland im Auftrag des Bundesministerium des Innern. Berlin: Bundesministerium des Inneren. Retrieved from http://www.bmi.bund.de/SharedDocs/Downloads/DE/Themen/OED_Verwaltung/ModerneVerwaltung/opengovernmen t.html

Kocka, J. (2004). Civil society from a historical perspective. European Review, 12(1), 65-79. Retrieved from http://ideas.repec.org/a/cup/eurrev/v12y2004i01p65-79_00.html

Koren, I., Guth, A., \& Klamma, R. (2013). Shared editing on the web: A classification of developer support libraries. In Collaborative Computing: Networking, Applications and Worksharing (Collaboratecom), 2013 9th International Conference Conference on (pp. 468-477). Austin, TX: IEEE.

Krafchik, W. (2013). Advocacy from the Inside: The Role of Civil Society. Innovating Government on a Global Stage. Sponsored Supplement to Stanford Social Innovation Review, 10.

Kriplean, T., Bonnar, C., Borning, A., Kinney, B., \& Gill, B. (2014). Integrating on-demand fact-checking with public dialogue. In Proceedings of the 17th ACM conference on Computer supported cooperative work \& social computing - CSCW '14 (pp. 1188-1199). New York, New York, USA: ACM Press. doi:10.1145/2531602.2531677

Kriplean, T., Morgan, J., Freelon, D., Borning, A., \& Bennett, L. (2012). Supporting reflective public thought with considerit. In Proceedings of the ACM 2012 conference on Computer Supported Cooperative Work - CSCW '12 (p. 265). New York, New York, USA: ACM Press. doi:10.1145/2145204.2145249

Lathrop, D., \& Ruma, L. (Eds.). (2010). Open Government: Collaboration, Transparency, and Participation in Practice. Beijing et al.: O'Reilly. Retrieved from http://github.com/oreillymedia/open_government

Lievrouw, L. (2011). Alternative and activist new media. Cambridge: Polity Press.

MacEachren, A. M., Cai, G., Sharma, R., Rauschert, I., Brewer, I., Bolelli, L., Shaparenko, B., Fuhrmann, S., Wang, H. (2005). Enabling collaborative geoinformation access and decision-making through a natural, multimodal interface. International Journal of Geographical Information Science, 19(3), 293-317. doi:10.1080/13658810412331280158

Maier-Rabler, U., \& Huber, S. (2011). "Open”: the changing relation between citizens, public administration, and political authority. eJournal of eDemocracy \& Open Government, 3(2), 182-191.

Malamud, C. (2010). By the People. In D. Lathrop \& L. Ruma (Eds.), Open Government: Collaboration, Transparency, and Participation in Practice (pp. 43-49). Beijing et al.: O'Reilly.

Masli, M., \& Terveen, L. G. (2014). Leveraging the contributory potential of user feedback. In Proceedings of the 17th ACM conference on Computer supported cooperative work \& social computing - CSCW'14 (pp. 956-966). New York, New York, USA: ACM Press. doi:10.1145/2531602.2531686

Meijer, A. J., Curtin, D., \& Hillebrandt, M. (2012). Open government: connecting vision and voice. International Review of Administrative Sciences, 78(1), 10-29. doi:10.1177/0020852311429533

Miller, C. C. (2006). A Beast in the Field: The Google Maps Mashup as GIS/2. Cartographica: The International Journal for Geographic Information and Geovisualization, 41(3), 187-199. doi:10.3138/j0l0-5301-2262-n779

Munson, J. P., \& Gupta, V. K. (2002). Location-based notification as a general-purpose service. In Proceedings of the 2nd international workshop on Mobile commerce - WMC '02 (p. 40). New York, New York, USA: ACM Press. doi:10.1145/570709.570713 
Nam, T. (2012). Citizens' attitudes toward Open Government and Government 2.0. International Review of Administrative Sciences, 78(2), 346-368. doi:10.1177/0020852312438783

Obermeyer, N. (1998). The evolution of public participation GIS. Cartography and Geographic Information Systems. Retrieved from http://www.tandfonline.com/doi/pdf/10.1559/152304098782594599

Orszag, P. R. (2009). Open Government Directive. Memorandum for the Heads of Executive Departments and Agencies. Executive Office of the President. Retrieved from http://www.whitehouse.gov/sites/default/files/omb/assets/memoranda_2010/m10-06.pdf

Parks, W. (1957). Open Government Principle: Applying the right to know under the Constitution. The George Washington Law Review, 26(1), 1-22.

Pollack, D. (2003). Zivilgesellschaft und Staat in der Demokratie. Forschungsjournal Neue Soziale Bewegungen, 16(2), 4658.

Quinlan, J. R. (1986). Induction of Decision Trees. Machine Learning, 1(1), 81-106. doi:10.1023/A:1022643204877

Quinlan, J. R. (1996). Improved use of continuous attributes in C4. 5. Journal of Artificial Intelligence Research. Retrieved from http://dl.acm.org/citation.cfm?id=1622742

Rinner, C. (1999). Argumaps for spatial planning. In R. Laurini (Ed.), First International Workshop on Telegeoprocessing (TeleGeo). Lyon.

Rinner, C. (2005). Computer Support for Discussions in Spatial Planning. In M. Campagna (Ed.), GIS for Sustainable Development (pp. 167-180). Taylor \& Francis.

Rinner, C. (2006). Argumentation Mapping in Collaborative Spatial Decision Making. In Collaborative Geographic Information Systems (pp. 85-102). IGI Global. doi:10.4018/978-1-59140-845-1.ch005

Rinner, C. (2007). Geovisualisierung zur räumlichen Entscheidungsunterstützung / Geovisualization to Support Spatial Decision-Making. Journal of Cartography and Geographic Information, 02, 85-92.

Rinner, C., \& Bird, M. (2009). Evaluating community engagement through argumentation maps - a public participation GIS case study. Environment and Planning B: Planning and Design, 36(4), 588-601. doi:10.1068/b34084

Robinson, D. G., Yu, H., Zeller, W. P., \& Felten, E. W. (2009). Government Data and the Invisible Hand. Yale Journal of Law \& Technology, 11, 159. Retrieved from http://papers.ssrn.com/abstract=1138083

Roth, R. (2003). Die dunklen Seiten der Zivilgesellschaft. Forschungsjournal Neue Soziale Bewegungen, 16(2), 59-73.

Rowe, G., \& Frewer, L. F. (2005). A Typology of Public Engagement Mechanisms. Science, Technology \& Human Values, 30(2), 251-290. doi:10.1177/0162243904271724

Safavian, S., \& Landgrebe, D. (1991). A survey of decision tree classifier methodology. IEEE Transactions on Systems, Man, and Cybernetics, 21(3), 660-674.

Semaan, B. C., Robertson, S. P., Douglas, S., \& Maruyama, M. (2014). Social media supporting political deliberation across multiple public spheres. In Proceedings of the 17th ACM conference on Computer supported cooperative work \& social computing - CSCW' 14 (pp. 1409-1421). New York, New York, USA: ACM Press. doi:10.1145/2531602.2531605

Sieber, R. (2006). Public Participation Geographic Information Systems: A Literature Review and Framework. Annals of the Association of American Geographers, 96(3), 491-507. doi:10.1111/j.1467-8306.2006.00702.x

Sieber, R. (2008). Participatory Geoweb: A research agenda. In Association of American Geographers Annual Meeting. Boston, MA.

Sifry, M. L. (2010). "You Can Be the Eyes and Ears": Barack Obama and the Wisdom of Crowds Micah. In D. Lathrop \& L. Ruma (Eds.), Open Government: Collaboration, Transparency, and Participation in Practice (pp. 117-124). Beijing et al.: O'Reilly.

Sohn, T., Li, K., Lee, G., Smith, I., Scott, J., \& Griswold, W. (2005). Place-lts: A study of Location-Based Reminders on Mobile Phones. In UbiComp 2005: Ubiquitous Computing (pp. 232-250). Springer. doi:10.1007/11551201_14 
Steinmann, R., Krek, A., \& Blasche, T. (2004). Analysis of online public participatory GIS applications with respect to the differences between the US and Europe. 24th Urban Data Management Symposium.

Sui, D. Z. (2004). GIS, Cartography, and the "Third Culture": Geographic Imaginations in the Computer Age. The Professional Geographer, 56(1), 62-72. doi:10.1111/j.0033-0124.2004.05601008.x

Sui, D. Z., \& Goodchild, M. (2011). The convergence of GIS and social media: challenges for GIScience. International Journal of Geographical Information Science, 25(11), 1737-1748. doi:10.1080/13658816.2011.604636

Sui, D. Z., \& Goodchild, M. F. (2001). GIS as media? International Journal of Geographical Information Science, 15(5), 387390. doi:10.1080/13658810110038924

Sui, D. Z., \& Goodchild, M. F. (2003). A tetradic analysis of GIS and society using McLuhan's law of the media. The Canadian Geographer/Le Géographe Canadien, 47(1), 5-17. doi:10.1111/1541-0064.02e08

Tapscott, D. (2010). Foreword. In D. Lathrop \& L. Ruma (Eds.), Open Government: Collaboration, Transparency, and Participation in Practice (pp. xv-xvii). Beijing et al.: O'Reilly.

United Nations Department of Economic and Social Affairs (UN DESA), \& Division for Public Administration and Development Managment (DPADM). (2013). The Guidelines on Open Government Data for Citizen Engagement (pp. 1-104). New York: United Nations. Retrieved from http://www.unpan.org/DPADM/EGovernment/OpenGovernmentDataandServices/tabid/1536/language/enUS/Default.aspx

Ware, C. (2004). Information Perception for Design (Second Edition). Morgan Kaufmann.

Weinstein, J., \& Goldstein, J. (2012). The Benefits of a Big Tent: Opening Up Government in Developing Countries. UCLA Law Review Discourse, 38, 39. Retrieved from http://www.uclalawreview.org/pdf/discourse/60-3.pdf

Yu, H., \& Robinson, D. G. (2012). The New Ambiguity of "Open Government." UCLA Law Review Discourse, 59, 178-208. doi:10.2139/ssrn.2012489

\section{About the Authors}

Thore Fechner is a PhD student at the Institute for Geoinformatics (ifgi), University of Münster, Germany and a software engineer specializing in spatial data infrastructures and eGovernment applications at con terra $\mathrm{GmbH}$. As a member of the Situated Computing Lab at ifgi, he is working on topics involving the design and evaluation of geospatial human computer interactions for citizen participation, Open Data and privacy. Thore can be contacted at t.fechner@uni-muenster.de.

Christian Kray is an Associate Professor at the University of Münster, Germany and Director at the Institute of Geoinformatics (ifgi). At ifgi, he also leads the Situated Computing Lab, which investigates evaluation methods for locationbased services as well as interaction methods for public displays and for map-based visualizations. His research interests include human computer interaction, spatial interaction and geoinformatics - in particular in the context of mobile and ubiquitous computing. Prior to joining ifgi, he was a senior lecturer at the School of Computing Science, Newcastle University, UK, and a postdoc at Lancaster University, UK. Chris received his Ph.D. (Dr.-Ing.) in Computer Science from Saarland University, Germany. Chris can be contacted at c.kray@uni-muenster.de. 